Proceedings of the 46th International School and Conference on the Physics of Semiconductors "Jaszowiec" 2017, Szczyrk

\title{
Accuracy of Effective Mass Equation for a Single and Double Cylindrical Quantum Dot
}

\author{
A. Mielnik-Pyszczorski*, K. Gawarecki and P. Machnikowski \\ Department of Theoretical Physics, Faculty of Fundamental Problems of Technology, Wrocław University of Science \\ and Technology, Wybrzeże Wyspiańskiego 27, 50-370 Wrocław, Poland

\begin{abstract}
In this contribution we study the accuracy of various forms of electron effective mass equation in reproducing spectral and spin-related features of quantum dot systems. We compare the results of the standard 8 band $\boldsymbol{k} \cdot \boldsymbol{p}$ model to those obtained from effective mass equations obtained by perturbative elimination procedures in various approximtions for a cylindrical quantum dot or a system of two such dots. We calculate the splitting of electronic shells, the electron $g$-factor and spin-orbit induced spin mixing and show that for a cylindrical dot the $g$-factor is reproduced very exactly, while for the two other quantities the effective mass equation is much less accurate.
\end{abstract}

DOI: 10.12693/APhysPolA.132.376

PACS/topics: 85.75.-d, 03.65.Yz

\section{Introduction}

The $\boldsymbol{k} \cdot \boldsymbol{p}$ method in the envelope function approximation $[1,2]$ has been very successful in modeling carrier states and kinetics, influence of strain, response to external fields and other properties of quantum dot (QD) systems [3-5]. While the 8 -band $\boldsymbol{k} \cdot \boldsymbol{p}$ model is an established standard of nanostructure modeling, in the cases when conduction band (cb) properties are of interest a simplified model including only the cb states is often applicable [6-8]. In common approaches, such a single-band or two-band (if spin is included) model is expressed by familiar Schrödinger- or Pauli-like equations for the envelope function, referred to as effective mass equations, leading to the popular "particle in a box" picture of confined carriers and, in many cases, allowing one to reduce mesoscopic semiconductor problems to textbook exercises in quantum mechanics. An important advantage of effective mass equations is their considerably reduced computational cost in the cases when numerical solution is required.

In our recent work [9] we presented a systematic derivation of cb effective mass equations from the 8-band $\boldsymbol{k} \cdot \boldsymbol{p}$ Hamiltonian. Various forms of such equations emerged as subsequent approximations within the framework of the Löwdin decoupling scheme [10]. We have assessed the accuracy of these approximations by calculating the splitting between the $s$ and $p$ states and the ground state Zeeman splitting in two models of a lens-shaped QD with different composition profiles. We showed that a quantitatively correct description of the lowest sector of the electron spectrum requires a self-consistent renormalization of the Hamiltonian parameters as well as accounting for cb non-parabolicity by self-consistently including terms

\footnotetext{
* corresponding author; e-mail:

adam.mielnik-pyszczorski@pwr.edu.pl
}

of higher order in the electron momentum. It turned out that an accurate value of the $g$ factor is obtained only after including the full structure of the valence band (vb) Hamiltonian in the equation, which yields a rather complicated equation that does not resemble the Pauli equation and does not even allow one to separate the kinetic and Pauli terms. An equation that is rigorously derived from the $\boldsymbol{k} \cdot \boldsymbol{p}$ theory strictly up to order $k^{2}$ (which may correspond to the most usual notion of an effective mass theory) quantitatively fails in all respects.

In this contribution we continue the previous analysis in two directions. First, we extend the study to another system geometry, a cylindrical QD, in order to gain broader evidence for our conclusions. Second, we study the performance of the efective mass equation in reproducing a much more sophisticated quantitative feature of the system: the spin-orbit induced anticrossing between two nominally opposite-spin Zeeman sublevels of ground states located in two coupled QDs. We show that the previous conclusions are essentially confirmed in the present case of a cylindrical QD, while for the subtle spin-orbit effect the effective mass approximation essentially captures only the order of magnitude of the resonant splitting, with intermediate approximations producing more accurate results than the nominally most exact one.

\section{Model and method}

We consider one or two cylindrical QDs placed on a wetting layer (WL). The height of the QDs is $4.2 \mathrm{~nm}$ and their radii are 9 and and $11.4 \mathrm{~nm}$ in the singleand double-QD case, respectively. The WL thickness is $0.6 \mathrm{~nm}$. In the double-QD system, the distance between the QDs is $10.2 \mathrm{~nm}$ (bottom to bottom). The dots are displaced along the in-plane direction (i.e., off-axis) by $6 \mathrm{~nm}$ in order to break the angular momentum conservation. The QDs and WL are composed of pure InAs and are embedded in a pure GaAs matrix. The computational box is $42 \times 42 \times 27 \mathrm{~nm}$. 
The general effective mass Hamiltonian can be written using two building elements [9]. The first one are $2 \times 6$ matrices $\mathcal{T}_{i}, i=1,2,3$, defined by writing the linear in $k$ part of the off-diagonal block of the 8-band $\boldsymbol{k} \cdot \boldsymbol{p}$ Hamiltonian $\left(H_{c v}=H_{6 c 8 v} \oplus H_{6 c 7 v}\right)$ in the form $H_{c v}=P \mathcal{T} \cdot \boldsymbol{k}$ (see Ref. [9] for the explicit definition). The second element is the $6 \times 6$ matrix (in the sense of the usual matrix notation for the multi-band $\boldsymbol{k} \cdot \boldsymbol{p}$ theory) $\mathcal{D}$, representing the local offset between the conduction and valence bands, keeping the full structure of the latter. This is defined as follows. First, one writes the cb block of the 8-band Hamiltonian, neglecting Zeeman terms, as $H_{6 c 6 c}=\chi_{c} \mathbb{I}_{2 \times 2}$. Then one defines $\mathcal{D}=\chi_{c} \mathbb{I}_{6 \times 6}-H_{v}$ where $H_{v}$ is the $6 \times 6$ valence band block of the Hamiltonian. Both $\chi_{c}$ and $H_{v}$ can depend on position but not on momenta, hence, depending on the chosen approximation, the $k$-dependent terms in these components are either neglected or replaced by self-consistently calculated average values in the state of interest. Here $\mathbb{I}_{n \times n}$ is the $n \times n$ unit matrix. Then, the effective mass Hamiltonian has the form [9]:

$$
\begin{aligned}
& \tilde{H}^{(2)}=\sum_{j l} k_{j} P \mathcal{T}_{j} \mathcal{D}^{-1} \mathcal{T}_{l}^{\dagger} P k_{l} \\
& \quad+\frac{1}{2} \sum_{j l}\left(P \mathcal{T}_{j} \mathcal{D}^{-1}\left[k_{j}, \chi_{c}^{\prime}\right] \mathcal{D}^{-1} \mathcal{T}_{l}^{\dagger} P k_{l}+\text { H.c. }\right) .
\end{aligned}
$$

Equation (1) can be taken at various levels of approximation by assuming different approximate forms for $\mathcal{D}$. As in Ref. [9], we consider the following series of approximations: (1) bulk approximation without strain, where $\mathcal{D}$ is diagonal and represents band offsets in a bulk crystal; (2) bulk approximation with strain, where we additionally include band shifts due to local strain via diagonal strainrelated terms; (3) semi-phenomenological approximation, where $\mathcal{D}$ is still diagonal and takes the constant value of the energy difference between the highest vb and lowest cb states in the QD obtained from the 8-band $\boldsymbol{k} \cdot \boldsymbol{p}$ model (to mimic the experimental fundamental transition energy), with an additional spin-orbit shift for the $\Gamma_{7 v}$ band; (4) off-diagonal approximation, where all the $k$-independent elements of $\chi_{c}$ and $H_{v}$ are included, thus yielding an equation that is strictly equivalent to the 8band model up to terms quadratic in $k$. (5) off-diagonal $+\left\langle k^{2}\right\rangle$ approximation, where the average values of the $k$-dependent terms in $\chi_{c}$ and $H_{v}$ are self-consistently included; (6) self-consistent effective mass equation, where the parameters of the original 8-band Hamiltonian are renormalized, which accounts for effects beyond perturbation theory (see Ref. [9] for details).

\section{Results}

In Fig. 1a we present the values of the $s-p$ energy separation for a single cylindrical QD obtained in the approximations defined above. As in the case of lens-shaped QD models discussed in Ref. [9], the overall accuracy of the effective mass theories is not impressive: effective models tend to overestimate this intraband excitation energy and even the most complicated approximation (6) yields

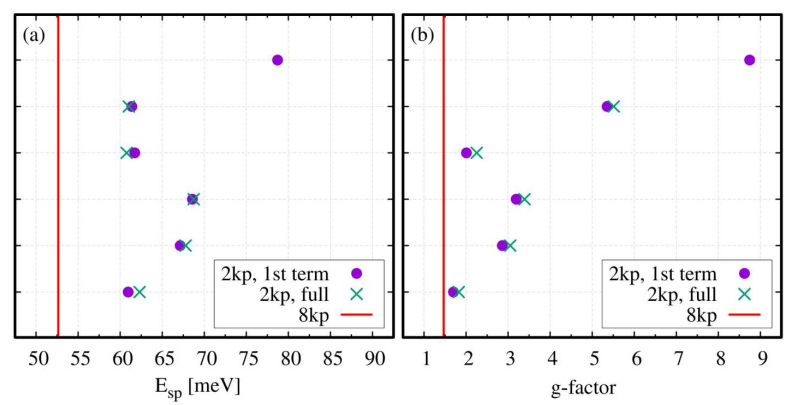

Fig. 1. Comparison of various effective mass approximations. (1) - bulk approximation without strain, (2) - bulk approximation with strain, (3) - semiphenomenological approximation, (4) - off-diagonal approximation, (5) - off-diagonal $+\langle k\rangle^{2}$ approximation, (6) - self-consistent effective mass equation. (a) Energy difference between the ground and first excited state in a single QD at zero magnetic field. (b) Ground state $g$-factor. In both figures the red line is the reference value from the 8-band $\boldsymbol{k} \cdot \boldsymbol{p}$ model, dots show the results obtained by taking only the first term of Eq. (1) into account and crosses show the values for the full Hamiltonian in a given approximation.

the result with $\approx 15 \%$ error, corresponding to $\approx 8 \mathrm{meV}$ absolute difference. Unlike the models of Ref. [9], where the most sophisticated approximation was the most accurate, here the standard approach with simple, diagonal $\mathcal{D}$ (approximation (2) or (3)) is able to reach the same accuracy.

Figure $1 \mathrm{~b}$ shows the results for the Landé factor of the ground state, obtained from the Zeeman splitting at $0.1 \mathrm{~T}$. For the most accurate approximation, the relative error is $20 \%$, which corresponds to only 0.29 in absolute value. The relative error is between the values obtained for two composition models in Ref. [9]. As in the QD models studied in that previous work, the full self-consistent model (6) yields the best results.

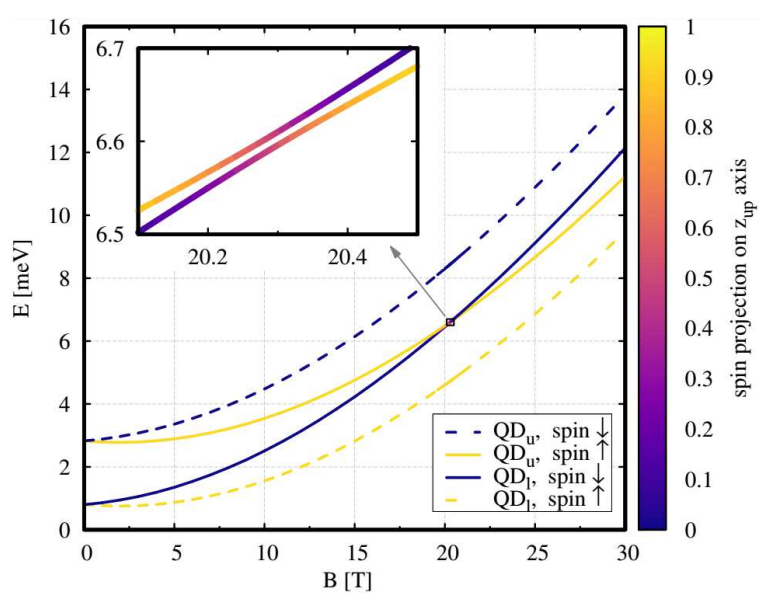

Fig. 2. Dependence of electronic states on the magnetic field in the DQD structure. 


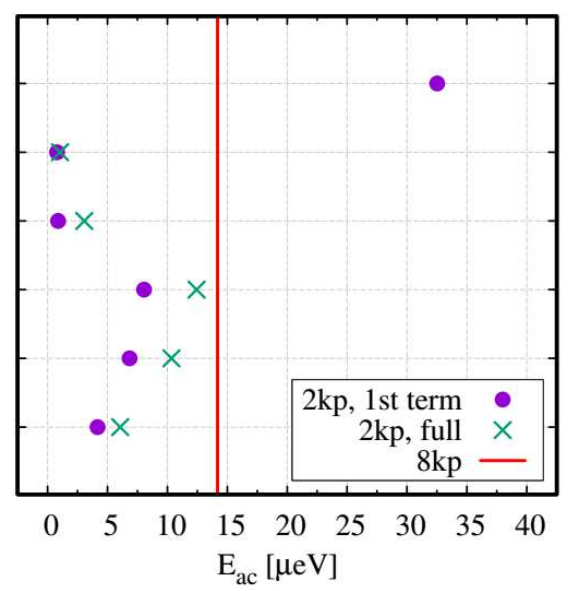

Fig. 3. Comparison of the spin-orbit anticrossing width according to different approximations. (1) — bulk approximation without strain, (2) - bulk approximation with strain, (3) - semi-phenomenological approximation, (4) — off-diagonal approximation, (5) — offdiagonal $+\langle k\rangle^{2}$ approximation, (6) - self-consistent effective mass equation.

Finally, we discuss spin mixing induced by spin-orbit effects resulting from structure inhomogeneity (the usual Dresselhaus terms are switched off in the models for the sake of clarity of the discussion). In a double-QD structure with broken axial symmetry coupling between two opposite Zeeman states belonging nominally to two diferent QDs becomes possible, which leads to an avoided crossing structure at the intersection of these two states (Fig. 2), thus yielding a spectral feature that allows one to quantify the sthrength of the spin-orbit effects. The results, shown in Fig. 3, indicate that even the most sophisticated equations are able to capture at most the order of magnitude of this effect. Moreover, the model involving self-consistent corrections, that was relatively reliable in the previous cases, produces less accurate results than the off-diagonal model without these corrections.

\section{Conclusions}

Our results indicate that a properly constructed effective mass equation with non-parabolicity corrections is able to quantitatively reproduce the system spectrum, including the Zeeman splitting, within 30\% accuracy. On the contrary, a more sophisticated feature related to spin-orbit coupling is not accurately reconstructed and tend to be considerably underestimated. This suggests in particular that effective mass models should be used with much care in the description of admixture-induced spin relaxation processes in self-assembled structures.

\section{References}

[1] L.C. Lew Yan Voon, M. Willatzen, The $k \cdot p$ Method, Springer, Berlin 2009.

[2] R. Winkler, Spin-Orbit Coupling Effects in TwoDimensional Electron and Hole Systems, Vol. 191 of Springer Tracts in Modern Physics, Springer, Berlin 2003.

[3] E. Kadantsev, P. Hawrylak, Phys. Rev. B 81, 045311 (2010).

[4] M. Doty, J. Climente, M. Korkusinski, M. Scheibner, A. Bracker, P. Hawrylak, D. Gammon, Phys. Rev. Lett. 102, 047401 (2009).

[5] P.-L. Ardelt, K. Gawarecki, K. Müller, A.A. Waeber, A. Bechtold, K. Oberhofer, J.J. Daniels, F. Klotz, M. Bichler, T. Kuhn, H.H. Krenner, P. Machnikowski, J.J. Finley, Phys. Rev. Lett. 116, 077401 (2016).

[6] J.M. Luttinger, W. Kohn, Phys. Rev. 97, 869 (1955).

[7] R. Dingle, W. Wiegmann, C.H. Henry, Phys. Rev. Lett. 33, 827 (1974).

[8] J. Maan, M. Altarelli, H. Sigg, P. Wyder, L. Chang, L. Esaki, Surf. Sci. 113, 347 (1982).

[9] A. Mielnik-Pyszczorski, K. Gawarecki, P. Machnikowski, arXiv:1706.04093, (2017).

[10] P.-O. Löwdin, J. Chem. Phys. 19, 1396 (1951). 